\title{
An EOQ Inventory Model with Fuzzy Deterioration Rate and Finite Production Rate
}

\author{
Neha Mishra ${ }^{1} \&$ Jitendra Kumar Soni ${ }^{2}$ \\ ${ }^{I}$ Department of Mathematics, $S$ R Group Of Institution, Jhansi \\ ${ }^{2}$ Department of Mathematics, $S$ R Group Of Institution, Jhansi (INDIA)
}

\begin{abstract}
In this paper, we have developed an economic production models with finite production rate and fuzzy deterioration rate. In the development of the model, lost of production quantity due to faulty machine aged machine, manufacturing defect etc. from the actual production quantity have also been taken into account. We developed the corresponding fuzzy model. The solution form minimizing the fuzzy cost function have been derived The sensitivity of the optimal solution with respect to the changes in the different parameter values is also discussed. all the inventory models have been developed under the assumption that the deterioration rate is constant or it is dependent on time.
\end{abstract}

\section{Introduction}

The basic EOQ model has the specific requirements of constant demand rate and lack of deterioration of the items in stock. Deterioration of an items can be defined as decay, evaporation obsolescence, loss of utility or marginal value of a commodity that results in the decreasing usefulness of the inventory from the original condition. Vegetables, meat, fertilizers, gasoline, different types of oils, medicines, mild etc. are examples of deteriorating items. Inventory models for deteriorating items has been studied by several researchers in recent decades. Gharese and Schrader[1]-[2] developed an EOQ model for items with an exponentially decaying inventory. An EOQ models for items with a variable rate of deterioration was discussed Covert and Philip[3] who used a two parameter weibull distribution for the time to deterioration. Covert and Philip[4] adopted a three parameters weibull distribution for the time to deterioration time. Mishra [5] formulated an inventory models with a variable rate of deterioration, a finite rate of deterioration, a finite rate of production. Several researcher like Cohen[6], Kang and Kim[7], Aggrawal and Jaggi [8-9]. Hui-Ming Wee [10], B.C. Giri [11] and K.S. Choudhuri [12] and San Chyi Chang[13] etc. developed economic production lot-size models with different assumption on the patterns on deterioration rate. Till now, all the inventory models have been developed under the assumption that the deterioration rate is constant or it is dependent on time. For solving the economic production equanimity, we always consider the demands rate, production rate and deterioration rate as constant in the crisp model. But, in the real life situation, these quantities will have little deviations from the exact value. The variables whose values are not crisp but uncertain in nature. Hence these variables should be treated as fuzzy variables. Yao and Lee [14] developed as economic order quantity model by considering order quantity as fuzzy and allowing shortages. The same authors Yao and Lee [15-16] developed another inventory model with fuzzy demand quantity and fuzzy production quantity.

In this, we have developed an economic production models with finite production rate and fuzzy deterioration rate. In the development of the model, lost of production quantity due to faulty machine aged machine, manufacturing defect etc. from the actual production quantity have also been taken into account. We developed the corresponding fuzzy model. The solution form minimizing he fuzzy cost function have been derived with the help of Zimmerman [17] and Kaufmann and Gupta[18] and the solution procedure is illustrated by one numerical example. The sensitivity of the optimal solution with respect to the changes in the different parameter values is also discussed.

\section{Notation And Assumption Of The Model}

The following notations have been used to develop the model.

1. $\mathrm{K}=$ Production Quantity per unit time.

2. $\mathrm{d}=$ Demand rate per unit time.

3. $\theta=$ deterioration rate of the on hand inventory per unit time.

4. $\tilde{\theta}=$ fuzzy deterioration rate of the on hand inventory per unit time.

5. $\phi=$ deterioration fraction of production, rate per unit time.

6. $\mathrm{C}_{1}=$ constant inventory holding cost per unit time

7. $\mathrm{r}=$ constant purchase cost of raw materials per unit item.

8. $\mathrm{b}=$ constant setup cost per order. 
9. $\mathrm{p}=$ constant selling price of the production

10. $\mathrm{t}_{1}=$ time at which production is stopped.

11. $\mathrm{t}_{2}=$ time at which inventory level reaches zero.

12. $\mathrm{T}=$ the whole period for the plan.

13. $\mathrm{q}_{1}=$ inventory level at any time $\mathrm{t}$, where $\mathrm{o} \leq \mathrm{t} \leq \mathrm{t}_{1}$

14. $\mathrm{q}_{2}=$ inventory level at any time $\mathrm{t}$, where $\mathrm{o} \leq \mathrm{t} \leq \mathrm{t}_{2}$

15. $\mathrm{q}=$ actual production quantity received per cycle.

16. $\mathrm{K}(1-\phi)=$ actual production rte per unit time.

\section{Assumptions Of The Model}

The following assumption are made in developing the model

1. Replenishment are instantaneous with constant lead time.

2. Shortage are not allowed

3. The membership function of the fuzzy deterioration rate $\bar{\theta}$

$$
\mu_{\bar{\theta}}(\theta)=\left\{\begin{array}{cl}
\frac{\theta-\theta_{1}}{\theta_{0}-\theta_{1}} & \text { for } \theta_{1} \leq \theta<\theta_{2} \\
\frac{\theta_{2}-\theta_{1}}{\theta_{2}-\theta_{0}} & \text { for } \theta_{0} \leq \theta<\theta_{2} \\
0 & \text { elsewhere }
\end{array}\right.
$$

Where $\theta_{1}, \theta_{0}$ and $\theta_{2}$ are positive numbers.

\section{Development Of The Crisp Model}

At time $t=0$ the production starts at the beginning of each cycle and continues upto time $t=t_{1}$. The actual production rate becomes less than original production reduce to faulty machine since some quantities deteriorate at the time of production.

The inventory accumulated during the production period $\mathrm{t}_{1}$ after meeting up demand during the period and deterioration reaches to the zero level at time $t=1_{2}$. Then the cycle repeat itself for total planning period $\mathrm{T}$.

The following differential equation gives the instantaneous states of $q_{1}$ and $q_{2}$ over the cycle $t_{1}$ \& $t_{2}$

$$
\begin{array}{lr}
\frac{d q_{1}}{d t}+\theta q_{1}=K(1-\phi)-d ; & 0 \leq \mathrm{t} \leq \mathrm{t}_{1} \\
\frac{d q_{2}}{d t}+\theta q_{2}=-d ; & \mathrm{t}_{1} \leq \mathrm{t} \leq \mathrm{t}_{2}
\end{array}
$$

With the condition

$\mathrm{q}_{1}(0)=0 ; \mathrm{q}_{1}\left(\mathrm{t}_{1}\right)=0, \mathrm{q}_{1}\left(\mathrm{t}_{1}\right)=\mathrm{q}_{2}\left(\mathrm{t}_{1}\right)$ and $\mathrm{q}_{2}\left(\mathrm{t}_{2}\right)=0$

Eq. (2... (2.3) is a linear differential equation with integrating factor (IF) given by

$$
\text { I.F. }=e^{\int \theta d t}=e^{\theta t}
$$

Its solution is given by

When

$$
\begin{aligned}
& e^{\theta t} \quad q_{1}(t)=\frac{(k(1-\phi)-d)}{\theta} e^{\theta t}+c \\
& t=0, \quad q_{1}(t)=0, \quad \text { we have } \\
& C=\frac{-(k(1-\phi)-d)}{\theta}
\end{aligned}
$$

$$
\begin{aligned}
& e^{\theta t} q_{1}(t)=\frac{(k(1-\phi)-d)}{\theta}\left(e^{\theta t}-1\right) \\
& \therefore q_{1}(t)=\frac{(k(1-\phi)-d)}{\theta}\left(1-e^{-\theta t}\right) \quad ; \quad 0 \leq \mathbf{t} \leq \mathbf{t}_{1}
\end{aligned}
$$


From equation (2.2), we get

$\frac{d q_{2}}{d t}+\theta q_{2}=-d$

Which is also a linear differential equation, its solution is given by

$\Rightarrow q_{2}(t)=\frac{d}{\theta}\left(e^{\theta\left(t_{2}-t_{1}\right)}-1\right)$

From equation (1.24) we have

$$
\begin{aligned}
q_{1}\left(t_{1}\right) & =\frac{(k(1-\varphi)-d)}{\theta}\left(1-e^{-\theta t_{1}}\right) \\
& =(k(1-\varphi)-d) \theta t_{1}\left(t_{1}-\frac{\theta t_{1}^{2}}{2}+\frac{\theta^{2} t_{1}^{3}}{6}\right)
\end{aligned}
$$

From equation (2.5), we have

$$
\begin{aligned}
q_{2}\left(t_{1}\right) & =\frac{d}{\theta}\left(e^{\theta\left(t_{2}-t_{1}\right)}-1\right) \\
& =\frac{d}{\theta}\left(1+\theta\left(\mathrm{t}_{2}-t_{1}\right)+\frac{\theta^{2}\left(t_{2}-t_{1}\right)^{2}}{2}-1\right) \\
& =\frac{d}{\theta}\left(\theta t_{2}-\theta t_{1}+\frac{\theta^{2}\left(t_{2}^{2}+t_{1}^{2}-2 t_{1} t_{2}\right)}{2}\right) \\
q_{1}\left(t_{1}\right) & =\frac{(k(1-\varphi)-d)}{\theta}\left(1-e^{-\theta t_{1}}\right) \\
& =\frac{(k(1-\varphi)-d)}{\theta}\left(1-e^{-\theta t_{1}}\right) \\
q_{2}\left(t_{1}\right) & =\frac{d}{\theta}\left(e^{\theta\left(t_{2}-t_{1}\right)}-1\right)=\frac{d}{\theta} \frac{\left(e^{\theta t_{2}}-e^{\theta t_{1}}\right)}{e^{\theta t_{1}}}
\end{aligned}
$$

given $q_{1}\left(t_{1}\right)=q_{2}\left(t_{1}\right)$

$$
t_{2}=\frac{q}{d}\left\{1+\frac{\theta t_{1}}{2}+\frac{\theta^{2} t_{1}^{2}}{C}\right\}
$$

Where $\mathrm{q}=\mathrm{k}(1-\varphi) \mathrm{t}_{1}$ is the actual production quantity during the period $\mathrm{t}_{1}$ Let $\mathrm{HC}$ denote the holding cost per cycle and is given by

$$
\begin{aligned}
H C & =C_{1}\left[\int_{0}^{t_{1}} q_{1}(t) d t+\int_{t_{1}}^{t_{2}} q_{2}(t) d t\right] \\
& =C_{1} \int_{0}^{t_{1}} \frac{(k(1-\varphi)-d)}{\theta}\left(1-e^{-\theta t}\right) d t+\int_{t_{1}}^{t_{2}} \frac{d}{\theta}\left\{e^{\theta\left(t_{2}-t\right)}-1\right\} d t \\
& =C_{1}\left[\int_{0}^{t_{1}}(k(1-\varphi)-d)\left(t-\frac{\theta t^{2}}{2}\right) d t+d \int_{t_{1}}^{t_{2}}\left(\left(t_{2}-t\right)+\theta \frac{\left(t_{2}-t\right)^{2}}{2}\right) d t\right]
\end{aligned}
$$




$$
\mathrm{HC}=C_{1}\left[(k(1-\varphi)-d)\left(\frac{t_{1}^{2}}{2}-\frac{\theta t_{1}^{3}}{6}\right)+d\left\{\frac{\left(t_{2}-t_{1}\right)^{2}}{2}+\frac{\theta\left(t_{2}-t_{1}\right)^{3}}{6}\right\}\right]
$$

Let LS denote the cost for loss of stock due to deterioration in each cycle and is given by

$$
\begin{aligned}
L S & =p\left[\theta\left\{\int_{0}^{t_{1}} q_{1}(t) d t+\int_{t_{1}}^{t_{2}} q_{2}(t) d t\right\}\right] \\
& =p\left\{k(1-\phi) t_{1}-d t_{2}\right\}
\end{aligned}
$$

Let LP denote the cost for loss of production quantity per cycle due to faulty machine and is given by

$\boldsymbol{L P}=\boldsymbol{r} \boldsymbol{k} \phi \boldsymbol{t}_{\boldsymbol{I}} \quad$ Also the number of cycles in the entire planning horizon $\mathrm{T}$ is $\mathrm{T} / \mathrm{t}_{2}$. Hence the total inventory cost, $\mathrm{TC}$ for the whole planning horizon $\mathrm{T}$ is given by

$$
\begin{aligned}
& T C=[H C+L C+L P+b] \cdot T / t_{2} \\
& =\left[\begin{array}{l}
C_{1}\left[\{K(1-\phi)-d\}\left(\frac{t_{1}^{2}}{2}-\frac{\theta t_{1}^{3}}{6}\right)+d\left\{\frac{\left(t_{2}-t_{1}\right)^{2}}{2}+\frac{\theta\left(t_{2}-t_{1}^{3}\right)}{6}\right\}\right] \\
+p\left\{\left(k(1-\phi) t_{1}-d t_{2}\right\}+r k \phi t_{1}+b\right.
\end{array}\right] \\
& =f_{1}(q)+\theta f_{2}(q)
\end{aligned}
$$

Where

$$
\begin{aligned}
& f_{1}(q)=\frac{C_{1} T}{2}\left\{1-\frac{d}{k(1-\phi}\right\} q+\left(b+\frac{r q \phi}{1-\phi}\right) \frac{d T}{q} \\
& f_{2}(q)=\frac{T}{2}\left\{\left(b+p q+\frac{r q \phi}{1-\phi}\right)+\frac{C_{1} q^{2}}{6}\left\{\frac{d}{k^{2}(1-\theta)^{2}}-\frac{1}{d}\right\}\right\}
\end{aligned}
$$

The model developed above gives the following particular cases

$$
\text { Case (i), If } \quad \phi \rightarrow 0, \quad k \rightarrow \infty, \quad \theta \rightarrow 0 \text {, we obtain }
$$

$$
T C=\frac{C_{1} q T}{2}+\frac{b d T}{q}
$$

This equation is same as the average total cost of a classical EOQ model with constant demand and no deterioration

CASE (II) when $\phi \rightarrow 0$, and $\mathrm{R}=\infty$

$T C=\frac{C_{1} q T}{2}+\frac{b d T}{q}+\frac{\theta T}{2}\left(b+p q-\frac{C_{1} q}{6}\right)$ demand.

Which is the classical EPQ (Economic Production Quality) model under deterioration and constant

CASE (III) when $\theta \rightarrow 0$, we get

$$
T C=\frac{C_{1} q T}{2}\left(1-\frac{d}{k}\right)+\frac{b d T}{q}+\frac{\theta T}{2}\left(1-\frac{d}{k}\right)\left\{b+p q-\left(1+\frac{d}{k}\right) \frac{C_{1} q^{2}}{6}\right\}
$$

Which is the classical EPQ (Economic Production Quality) model under deterioration and constant demand.

CASE (IV) : For $\phi \rightarrow 0, \quad \theta \rightarrow 0$, we get 


$$
\begin{aligned}
& f_{1}(q)=\frac{C_{1} T}{2}\left(1-\frac{d}{k}\right) q+b \cdot \frac{d T}{q} \\
& T C=\frac{C_{1} q T}{2}\left(1-\frac{d}{k}\right)+b \cdot \frac{d T}{q}
\end{aligned}
$$

Which is same as obtained by Lee \& Yao (1998).

\section{Development Of Fuzzy Model}

Earlier authors have assumed that the deterioration rate is constant fraction of the on hand inventory level in the development of Economic Order Quantity models. But in real world it is not always easy to determine the deterioration rate exactly but in most cases it is uncertain in nature. The Economic Order Quantity model have been developed with the assumption that deterioration rate is a fuzzy number $\tilde{\theta}$ instead of considering deterioration rate as constant.

Thus the function of (2.12) can be redefined as

$$
\text { Min TC }=f_{I}(q)+\tilde{\theta} f_{2}(q) \text { where } q \geq 0
$$

Where Wavy bar $\sim$ represents the fuzzification of the parameters.

We express the fuzzy deterioration rate $\tilde{\theta}$ as the normal triangular fuzzy number $\left(\theta_{1}, \theta_{0}, \theta_{2}\right)$

Suppose the membership function of the fuzzy deterioration rate $\tilde{\theta}$ is as follows :-

$$
\mu_{\widetilde{\theta}}(\theta)=\left\{\begin{array}{c}
\frac{\theta-\theta_{1}}{\theta_{0}-\theta_{1}} \text { for } \theta_{1} \leq \theta \leq \theta_{0} \\
\frac{\theta_{2}-\theta}{\theta_{2}-\theta_{0}} \text { for } \theta_{0} \leq \theta \leq \theta_{2} \\
0 \quad \text { elsewhere }
\end{array}\right.
$$

Where $\theta_{1}, \theta_{0}$ and $\theta_{2}$ are positive values and $0 \leq \theta_{1}<\theta_{0}<\theta_{2}$

The centroid of $\mu_{\tilde{\theta}}(\theta)$ is given by

$\mathrm{M}_{0}\left(\theta_{1}, \theta_{0}, \theta_{2}\right)=\left(\theta_{1}+\theta_{0}+\theta_{2}\right) / 3$

For any positive numbers $\mathrm{C}_{1}, \mathrm{~d}, \mathrm{~b}, \mathrm{~T}, \varphi, \mathrm{p}, \mathrm{r}, \mathrm{k}$ and every fixed value of $\mathrm{q}>0$.

Let $\mathrm{G}_{\mathrm{q}}(\theta)$ denotes the total cost function TC ( $\left.\mathrm{q}, \theta\right)$ for $\mathrm{q}, \theta$.

$\mathrm{G}_{\mathrm{q}}(\theta)=\mathrm{f}_{1}(\mathrm{q})+\theta \mathrm{f}_{2}(\mathrm{q})=\mathrm{Z}$.

Then we have

$$
\begin{aligned}
& \theta=\frac{z-f_{1}(q)}{f_{2}(q)} \quad \text { for } f_{2}(q) \neq 0 \\
& \Rightarrow z \geq f_{1}(q)=z_{1}(q)
\end{aligned}
$$

By extension principle, the membership function for fuzzy cost function $\mathrm{G}_{\mathrm{q}}(\tilde{\theta})$ is given by

$$
\begin{array}{ll}
\mu_{G_{q}(\widetilde{\theta})}(z)=\operatorname{Sup}_{\theta \in G^{-1}(z)} \mu_{\widetilde{\theta}} & \text { if } G_{q}^{-1}(z) \neq \phi \\
=0 & \text { if } G_{q}^{-1}(z)=\phi
\end{array}
$$

Thus if $\mathrm{z} \geq \mathrm{z}_{1}(\mathrm{q})$ then

Now let

$$
\begin{aligned}
& \mu_{G_{q}(\tilde{\theta})}(z)=\mu_{\tilde{\theta}}(\theta) \\
& =0 \quad \text { else. }
\end{aligned}
$$


$C_{j}(q)=f_{1}(q)+\theta_{j} f_{2}(q)$

So $\quad C_{j}(q)-z_{1}(q)=\theta_{j} f_{2}(q) \geq 0$

$\Rightarrow z_{1}(q) \leq C_{j}(q)$

and $C_{i}(q)-C_{j}(q)=\left(\theta_{i}-\theta_{j}\right) f_{2}(q) \quad$ for $j=1,0,2$

and $\mathrm{O} \leq \theta_{1}<\theta_{0}<\theta_{2}$

Further

$\theta_{\mathrm{i}} \geq \theta_{j} \Leftrightarrow C_{i}(q) \geq C_{j}(q)$

for $(\mathrm{i}=0,2, \mathrm{j}=1)$ or $(\mathrm{i}$
and $\quad \theta_{i} \leq \theta_{j} \Leftrightarrow C_{i}(q) \geq C_{j}(q)$

for $(i=0,1, j=2)$ or $(i=1, j=0)$

$$
\mathbf{P}=\int_{-\infty}^{\infty} \mu_{G_{q}(\tilde{\theta})}(z) d z
$$

and

$$
R=\int_{-\infty}^{\infty} \mu_{G_{q}(\tilde{\theta})}(z) d z
$$

The centroid for $\mu_{G_{q}(\tilde{\theta})}(z)$ is given by R/P which is the estimate of the total cost.

We have $\mathrm{Z}_{1}(\mathrm{q}) \leq \mathrm{C}_{1}(\mathrm{q})<\mathrm{C}_{0}(\mathrm{q})<\mathrm{C}_{2}$ (q)

Using the condition (2.18) to (2.19a)

The membership function of $G_{q}(\tilde{\theta})$ can be defined as

$$
\begin{array}{ll}
\mu_{G_{q}(\tilde{\theta})}(z)=\frac{z-f_{1}(q)-\theta_{1} f_{2}(q)}{\left(\theta_{0}-\theta_{1}\right) f_{2}(q} & \text { for } \mathrm{C}_{1}(\mathrm{q}) \leq \mathrm{z} \leq \mathrm{C}_{0}(\mathrm{q}) \\
=\frac{f_{1}(q)+\theta_{2} f_{2}(q)-2}{\left(\theta_{0}-\theta_{1}\right) f_{2}(q)} & \text { for } C_{0}(q) \leq z \leq C_{2}(q)
\end{array}
$$

$=0, \quad$ else where

Therefore $\mathrm{P}$ and $\mathrm{R}$ can be written as

$$
\begin{aligned}
& P=\frac{1}{\left(\theta_{0}-\theta_{1}\right) f_{2}(q)} \int_{C_{1}(q)}^{C_{0}(q)}\left(z-f_{1}(q)-\theta_{1} f_{2}(q)\right) d z \\
& +\frac{1}{\left(\theta_{2}-\theta_{0}\right) f_{2}(q)} \int_{C_{1}(q)}^{C_{2}(q)}\left\{f_{1}(q)+\theta_{2} f_{2}(q)-z\right\} d z \\
& =\frac{\left(\theta_{2}-\theta_{1}\right) f_{2}(q)}{2}
\end{aligned}
$$




$$
\begin{aligned}
& R=\frac{1}{\left(\theta_{0}-\theta_{1}\right) f_{2}(q)} \int_{C_{1}(q)}^{C_{0}(q)} z\left\{z-f_{1}(q)-\theta_{1} f_{2}(q)\right\} d z \\
& +\frac{1}{\left(\theta_{2}-\theta_{0}\right) f_{2}(q)} \int_{C_{1}(q)}^{C_{2}(q)}\left\{f_{1}(q)-\theta_{2} f_{2}(q)-z\right\} d z \\
& =\left(\theta_{2}-\theta_{1}\right) f_{2}(q) \cdot\left\{3 f_{1}(q)+\left(\theta_{0}+\theta_{1}+\theta_{2}\right) f_{2}(q)\right\} / 6
\end{aligned}
$$

After evaluating the integral of $\mathrm{R}$ and $\mathrm{P}$ and with simple algebra, we obtain the centroid of $\mu_{G_{q}(\tilde{\theta})}(z)_{\text {as }}$

$$
\frac{R}{P}=f_{1}(q)+\left(\theta_{1}+\theta_{0}+\theta_{2}\right) f_{2}(q) / 3
$$

\section{Optimal Solution Of The Inventory Model}

For given $\theta_{0}$, we have to find $\left(\theta_{1}^{*}, \theta_{2}^{*}, q^{*}\right)$ such that the centroid of the fuzzy total cost is minimal i.e.

$$
\operatorname{Min} \frac{R\left(\theta_{1}, \theta_{2}, q\right)}{P\left(\theta_{1}, \theta_{2}, q\right)}=\frac{R\left(\theta_{1}^{*}, \theta_{2}^{*}, q^{*}\right)}{P\left(\theta_{1}^{*}, \theta_{2}^{*}, q^{*}\right)}
$$

Since the equation (2.23) is a highly non linear. It is difficult to minimize the cost function analytically. We have used the standard software package LINGO for minization of the cost function (2.23).

Then we regards as the Economic production quantity.

Also

$$
\hat{\theta}_{0}=\left(\theta_{1}^{*}+\theta_{0}^{*}+\theta_{2}^{*}\right) / 3
$$

Is the centroid of the normal fuzzy triangular number $\left(\theta_{1}^{*}, \theta_{0}, \theta_{2}^{*}\right)$.

\section{Numerical Illustration Of The Model}

Let,

The following numerical example has been considered to illustrate the Inventory model.

$\begin{array}{lll}\mathrm{C}_{1} & = & 10 \\ \mathrm{~d} & = & 2 \\ \mathrm{~b} & = & 500 \\ \mathrm{~T} & = & 40 \\ \mathrm{p} & = & 3 \\ \mathrm{r} & = & 1 \\ \mathrm{k} & = & 10 \\ \theta & = & 0.003 \\ \phi & = & .005\end{array}$

In appropriate units.With these parameter values the solution of the equation (2.24).

Using the software LINGO are obtains as

$$
\begin{aligned}
& q^{*}=15.89, \theta_{1}^{*}=0.001, \theta_{2}^{*}=0.005 \\
& \text { and } \frac{\mathrm{R}}{\mathrm{P}}=5071.059
\end{aligned}
$$


Thus $\theta_{0}^{*}=0.003$ if $\quad \theta_{0}=0, \theta_{1}=0, \theta_{2}=0$

Then $q^{*}=15.821$

and $\quad \frac{\mathrm{R}}{\mathrm{P}}=5056.866$

\section{Sensitivity Analysis Of The Model}

We have examined the sensitivity of each of the decision variables $\mathrm{q}^{*}, \theta_{1}{ }^{*}, \theta_{2}{ }^{*}$, using he numerical example and the centroid of the fuzzy total cost R/P to changes in each of the eight parameters e,d,b,p,r,k, $\theta_{0}$ and $\varphi$.

Where each parameters has been varied from $-50 \%$ to $+50 \%$ keeping other parameters constant. The results obtained are displaced in table 1.1

Table 1.1 : Effect of changes in parameters on the decision variables.

\begin{tabular}{|l|l|l|l|l|l|}
\hline Parameters & Changes $(\%)$ & $\mathrm{q}^{*}$ & $\theta_{1}{ }^{*}$ & $\theta_{2}{ }^{*}$ & $\begin{array}{l}\text { Total } \\
(\mathrm{R} / \mathrm{P})\end{array}$ \\
\hline $\mathrm{C}$ & +50 & 12.964 & 0.00000 & 0.00600 & 6207.063 \\
& +20 & 14.501 & 0.00000 & 0.00590 & 5553.479 \\
& -20 & 17.774 & 0.00097 & 0.00503 & 4537.498 \\
& -50 & 22.507 & 0.00000 & & 3590.985 \\
\hline $\mathrm{D}$ & +50 & 22.809 & 0.00156 & 0.00444 & 5801.410 \\
& +20 & 17.859 & 0.00300 & 0.00300 & 5411.154 \\
& -20 & 13.872 & 0.00091 & 0.00508 & 4650.951 \\
& -50 & 10.601 & 0.00028 & 0.00572 & 3811.510 \\
\hline $\mathrm{B}$ & +50 & 19.483 & 0.00043 & 0.00557 & 6213.923 \\
& +20 & 17.415 & 0.00300 & 0.00300 & 5556.267 \\
& -20 & 14.205 & 0.00000 & 0.00599 & 4534.615 \\
& -50 & 11.220 & 0.00300 & 0.00300 & 3583.439 \\
\hline $\mathrm{P}$ & +50 & 15.887 & 0.00108 & 0.00492 & 5072.201 \\
& +20 & 15.889 & 0.00106 & 0.00494 & 5071.516 \\
& -20 & 15.892 & 0.00104 & 0.00493 & 5070.602 \\
& -50 & 15.894 & 0.00101 & 0.00499 & 5069.926 \\
\hline $\mathrm{R}$ & & & & & \\
& +50 & 15.890 & 0.00105 & 0.00495 & 5071.262 \\
& +20 & 15.890 & 0.00105 & 0.00495 & 5071.140 \\
& -20 & 15.890 & 0.00105 & 0.00495 & 5070.978 \\
\hline $\mathrm{K}$ & -50 & 15.890 & 0.00105 & 0.00495 & 5070.856 \\
\hline & +50 & 15.256 & 0.00300 & 0.00300 & 5281.584 \\
& +20 & 15.564 & 0.00107 & 0.00493 & 5177.367 \\
& -20 & 16.421 & 0.00099 & 0.00500 & 4907.379 \\
\hline$\theta_{0}$ & -50 & 18.399 & 0.00037 & 0.00563 & 4380.649 \\
\hline & +50 & 15.925 & 0.00158 & 0.00742 & 5048.119 \\
& +20 & 15.904 & 0.00126 & 0.00594 & 5073.886 \\
& -20 & 15.876 & 0.00240 & 0.00240 & 5068.228 \\
& -50 & 15.856 & 0.00150 & 0.00150 & 5063.975 \\
\hline \multirow{2}{*}{} & +50 & 15.895 & 0.00300 & 0.00300 & 5069.640 \\
& +20 & 15.892 & 0.00105 & 0.00495 & 5070.492 \\
& -20 & 15.888 & 0.0105 & 0.00495 & 5071.624 \\
& -50 & 15.885 & 0.00300 & 0.00300 & 5072.470 \\
\hline
\end{tabular}

We see from table 1.1 that all he decision variables are sensitive to changes in $C_{1}$ total cost decreases from $29 \%$ for a $50 \%$ decrease in $\mathrm{C}_{1}$ and increases by $22 \%$ for a $50 \%$ increase in $\mathrm{C}_{1}$. All the decision variables are sensitive to changes in $\mathrm{d}$.

For a $50 \%$ increases, q and total cost increases by $31 \%$ and $14 \%$ respectively. On the other hand when $\mathrm{d}$ is reduced by $50 \%$, q and total cost are decreased by $33 \%$ and $24 \%$ respectively. For changes in the parameters 
b, we observe that $q$ and total cost are sensitive whereas $\theta_{1}$ and $\theta_{2}$ are very less sensitive. The decision variable $q$ and total cost increases and decreases by $22 \%$ and $29 \%$ respectively, for a $50 \%$ increment and decrement in b.

\section{Conclusion}

In the inventory model, in order to solve the economic production quantity precycle we always treat the deterioration rate as constant. But, in the real situation, this quantity probably will have little disturbances. Therefore, we should fuzzily this quantity to solve the economic production quantities it the fuzzy sense. In this article, a perishable inventory model with finite production rate, constant demands rate and fuzzy deterioration rate is developed for finite planning horizon. Also loss of production incurred due to faulty/aged machines have been taken into account by considering a fraction of production rate deteriorates per unit time. The theory for minimizing the fuzzy total cost function is developed by constructing the membership function of the cost function. The solution procedure is illustrated with analysis of the parameters of the system is carried out. It is observed that $\mathrm{c}_{1}, \mathrm{~b}, \mathrm{~d}$, and $\mathrm{k}$ are sensitive parameters of the system. The other parameters have no significant sensitivity.

\section{References}

[1] Ghare P.M. and Schrader G.F. An inventory model for exponentially deteriorating items. Journal of industrial Engineering.,14: 238-243,(1963)

[2] Ghare P.M. and Schrader, G.P., “A model for exponentially decaying inventory”, Journal of Industrial Engineering ,14: 238-243, (1963)

[3] Convert R.P. and Philip G.C., An EOQ model for items with Weibull distribution deterioration. AIIE Trans, 5: 323-326, (1973)

[4] Covert, R.P. and Philip, G.C.,An EOQ model items with weibull distribution deterioration” A.I.T.E. Transactions, 5:323326,(1973)

[5] Misra R.B. : Optimum production lot-size model for a system with deteriorating inventory. International Journal of Production Research,13:495-505. (1976)

[6] Cohen, M.A.: Joint pricing and ordering policy for exponentially decaying inventory with known demand. Naval Research Logistics Quarterly, 24: 257-268, (1977)

[7] Kang. S. and Kim. I: "A study on the price and production level of the deteriorating inventory system". International Journal Production Research,21:449-460, (1983)

[8] Aggarwal S.P. and Jaggi C.K. “Ordering policies of deteriorating items under permissible delay in payments", Journal of operation research society, 46:658-662, (1995)

[9] Aggrawal S.P. and Jaggi C.K.: Ordering policy for decaying inventory". International Journal of system of system Sciences, 20:151-155, (1989)

[10] Wee, Hui-Ming : "A replenishment policy for items with a price dependent demand and varying rate of deterioration". Production Planning and Control. 8:No. 5, 494-499, (1997)

[11] Giri, B.C. and Choudhary, K.S.: Deterministics models of perishable with stock dependent demand rate and non-linear holding cost". European Journal of Operational Research, 105: 467-474, (1998)

[12] Chakraborty T \& Chaudhuri K.S. :An EOQ model for items with Weibull distribution deterioration shortages and trended demand: An extension of Philips model, computer's and operations research . 25 7/8: 649-657, (1998)

[13] Chung, K.H. "Inventory control and trade credit revised" Journal of operational research society, 40: 495-498, (1989)

[14] Yao J.S. and Lee H.M.: "Economic production quantity for fuzzy demand quantity and fuzzy production quantity". European journal of operation research, 109: (2003-2011).

[15] Yao, J.S. and Lee H.M., “" Fuzzy Inventory with backorder for fuzzy order quantity" information sciences, 93:203-319, (1996)

[16] Yao, J.S., Chang S.C. and Su. J.S. "Fuzzy inventory without back order for fuzzy order quantity and fuzzy total demand quantity".Computer and operation research, 27:935-962, (2000)

[17] Zimmerman H.J.: Fuzzy set theory and its application, $2^{\text {nd }}$ Revised ed. Kluwer Academic Publisher Dordrecht. (1991)

[18] Kaufmann, A and Gupta M.M.: Introduction to Fuzzy Arithmetic Theory and Application” Van Nostrand Ranhold, New York. (1991) 